\title{
THE EQUIVALENCE OF ZERO SPAN AND ZERO SEMISPAN
}

\author{
JAMES FRANCIS DAVIS
}

\begin{abstract}
In this paper we introduce the idea of the symmetric span of a continuum, and show that continua with zero symmetric span are in class $W$ Continua with zero span have zero symmetric span, but the converse does not hold. We also show that if every subcontinuum of the continuum $M$ is in class $W$ then the span of $M$ and the semispan of $M$ agree. These results are then applied to show that the properties of having zero span and of having zero semispan are equivalent.
\end{abstract}

1. Introduction. The concept of the span of a metric space was introduced by A. Lelek in 1964 [7]. Since that time it has become an important concept in continuum theory, particularly in regard to chainability of continua. It is known, for instance [7, p. 210], that chainable continua have zero span. It is an open question as to whether or not continua which have zero span are chainable (see [8, Problem 1, p. 93; 5, Question 7, p. 331]). In 1977 Lelek introduced the concept of semispan [9] by relaxing a condition in his original definition.

In this paper we introduce and investigate symmetric span, another idea related to span. In particular, we show that continua with zero symmetric span are in class $W$. We also investigate the relationship between semispan and span, and show that these measures agree for a class of continua including those having zero span.

2. Definitions. A continuum is a nondegenerate connected metric space. A mapping is a continuous function. Suppose $M$ is a continuum with metric $d$. Denote the first and second projections of $M \times M$ onto $M$ by $\pi_{1}$ and $\pi_{2}$, respectively. The span of $M$ (respectively, the semispan of $M$ ), denoted by $\sigma(M)$ (respectively, $\sigma_{0}(M)$ ), is the least upper bound of the set of all numbers $\varepsilon$ for which there exists a subcontinuum $Z$ of $M \times M$ such that $\pi_{2}(Z)=\pi_{1}(Z)$ (respectively, $\left.\pi_{2}(Z) \subset \pi_{1}(Z)\right)$ and $d(x, y) \geqslant \varepsilon$ for each $(x, y)$ in $Z$.

If $\mathcal{G}$ is a collection of sets, denote the union of the members of $\mathcal{G}$ by $\mathcal{G} *$.

3. Symmetric span. As an example of a continuum with positive span, consider the unit circle in the complex plane, $S^{1}=\{z: z \in C$ and $|z|=1\}$. To see that $\sigma\left(S^{1}\right)>0$

Received by the editors December 1, 1982 and, in revised form, May 19, 1983; presented to the Society January 8, 1981 in San Francisco.

1980 Mathematics Subject Classification. Primary 54F20, 54C10; Secondary 54F50, 54H25.

Key words and phrases. Span, semispan, weakly confluent mappings, fixed point property.

'The paper is part of the author's dissertation, written under the direction of W. T. Ingram, and presented to the Faculty of the University of Houston in partial fulfillment of the requirements for the $\mathrm{Ph}$. D. degree, August 1981. 
let $Z=\left\{(z,-z): z \in S^{1}\right\}$. If $(x, y)$ is in $Z$ then $d(x, y)=2$. To see that $\pi_{1}(Z)=$ $\pi_{2}(Z)$ one need only observe that if $(x, y)$ is in $Z$ then $(y, x)$ is in $Z$. Continua which have the last property of the continuum $Z$ above are often constructed in studying the span of examples (see [3] for instance). This motivates the following definition.

If $X$ is a set and $A \subset X \times X$, then $A^{-1}=\{(y, x):(x, y) \in A\}$, and $A$ is said to be symmetric if $A=A^{-1}$. Suppose $M$ is a continuum with metric $d$. The symmetric span of $M$, denoted by $s(M)$, is the least upper bound of the set of all numbers $\varepsilon$ for which there exists a symmetric subcontinuum $Z$ of $M \times M$ such that $d(x, y) \geqslant \varepsilon$ for each $(x, y)$ in $Z$.

As observed before, if $M$ is a continuum and $Z$ is a subcontinuum of $M \times M$ such that $Z=Z^{-1}$, then $\pi_{1}(Z)=\pi_{2}(Z)$. A straightforward application of this observation proves the following theorem giving the relationship between the span and the symmetric span of a continuum.

THEOREM 1. If $M$ is a continuum, then $0 \leqslant s(M) \leqslant \sigma(M)$. Consequently if $\sigma(M)=0$ then $s(M)=0$.

Howard Cook has shown that the dyadic solenoid has a property (actually, (3) of Theorem 2 below) that is equivalent to having symmetric span equal to zero. Thus the last implication in Theorem 1 cannot be reversed.

Some useful and easily established equivalences of the property of having zero symmetric span are given in the following theorem.

THEOREM 2. Suppose $M$ is a continuum. Then the following are equivalent.

(1) $s(M)=0$.

(2) If $Z$ is a subcontinuum of $M \times M$ such that $Z=Z^{-1}$ then $Z$ intersects $\Delta_{M}$.

(3) If $Z$ is a subcontinuum of $M \times M$ and $Z$ intersects $Z^{-1}$, then $Z$ intersects $\Delta_{M}$.

THEOREM 3. If $M$ is a continuum and $s(M)=0$ then $M$ is atriodic and hereditarily unicoherent, and therefore hereditarily irreducible.

Proof. First suppose that $M$ contains a triod. Then there are subcontinua $H_{1}, H_{2}$ and $H_{3}$ of $M$ such that $H_{i} \cap H_{j}$ is a common continuum for all distinct pairs of $i$ and $j$, and $H_{i} \not \subset H_{j} \cup H_{k}$ if $i \neq j$ and $i \neq k$. Let $p_{i}$ be a point in $H_{i}-\left(H_{1} \cap H_{2} \cap H_{3}\right)$ for $i=1,2,3$. Defining $C_{i}=\left\{p_{i}\right\} \times\left(H_{j} \cup H_{k}\right)$ for $\{i, j, k\}=\{1,2,3\}$, let $Z=$ $\cup_{i=1}^{3}\left(C_{i} \cup C_{i}^{-1}\right)$. Then $Z$ is a subcontinuum of $M \times M$ and $Z=Z^{-1}$. However $Z$ does not intersect $\Delta_{M}$. This involves a contradiction with Theorem 2(2).

Now suppose that $M$ contains a nonunicoherent subcontinuum. Then there are subcontinua $H$ and $K$ of $M$ such that $H \cap K$ is not connected. Let $p$ and $q$ be points of $H \cap K$ which are in different components of $H \cap K$. Let $V_{p}$ and $V_{q}$ be mutually exclusive open sets containing $p$ and $q$, respectively, such that $H \cap K \subset V_{p} \cup V_{q}$. Let $T_{H}$ and $T_{K}$ be the closures of the components of $V_{p} \cap H$ and $V_{p} \cap K$, respectively, which contain $p$. By [10, Theorem 50, p. 18] the continua $T_{H}$ and $T_{K}$ contain points $r_{H}$ and $r_{K}$, respectively, which are not in $V_{p}$. Let

$$
C=\left(\{q\} \times T_{H}\right) \cup\left(K \times\left\{r_{H}\right\}\right) \cup\left(\left\{r_{K}\right\} \times H\right) \cup\left(T_{K} \times\{q\}\right)
$$


and $Z=C \cup C^{-1}$. It is easily seen that $Z=Z^{-1}$ and that $Z$ does not intersect $\Delta_{M}$. Hence we have a contradiction again with Theorem 2(2).

Thus $M$ is atriodic and hereditarily unicoherent. It follows from [11, Theorem 3.2, p. 456] that $M$ is hereditarily irreducible.

4. Symmetric span and class $W$. A mapping $f$ from a continuum $X$ onto a continuum $M$ is weakly confluent provided that for each subcontinuum $H$ of $M$, there is a component $K$ of $f^{-1}(H)$ such that $f(K)=H$. A continuum $M$ is in class $W$ provided that for each mapping $f$ from a continuum onto $M, f$ is weakly confluent.

THEOREM 4. If $M$ is a continuum and $s(M)=0$ then $M$ is in class $W$.

Proof. Suppose $M$ is a continuum which is not in class $W$, and that $s(M)=0$. Suppose $X$ is a continuum and $f$ is a mapping of $X$ onto $M$ which is not weakly confluent. Then there is a subcontinuum $C$ of $M$ such that no component of $f^{-1}(C)$ is mapped by $f$ onto $C$. Since $s(M)=0$, by Theorem 3, $C$ is irreducible between some two points, $p$ and $q$. Then no component $K$ of $f^{-1}(C)$ has the property that both $p$ and $q$ are in $f(K)$.

Let $\mathcal{H}_{p}=\left\{K: K\right.$ is component of $f^{-1}(C)$ and $\left.p \in f(K)\right\}, \mathcal{H}_{q}=\{K: K$ is a component of $f^{-1}(C)$ and $\left.q \in f(K)\right\}, A=\mathcal{H}_{p}^{*}$ and $B=\mathcal{H}_{q}^{*}$. It follows that $A$ and $B$ are mutually exclusive; otherwise a component of $f^{-1}(C)$ would contain both $p$ and $q$.

We will now show that $A$ and $B$ are closed. Suppose $x_{1}, x_{2}, x_{3}, \ldots$ is a sequence of points in $A$ with limit $y$. For each positive integer $i$, let $K_{i}$ be the component of $f^{-1}(C)$ which contains $x_{i}$, and let $z_{i}$ be a point of $K_{i}$ such that $f\left(z_{i}\right)=p$. There exists a subsequence $K_{n(1)}, K_{n(2)}, K_{n(3)}, \ldots$ of $K_{1}, K_{2}, K_{3}, \ldots$ with a sequential limiting set, $K_{0}$, which is a continuum [10, Theorems 58, 59, pp. 23-24]. There exists a subsequence $z_{n(i(1))}, z_{n(i(2))}, z_{n(i(3))}, \ldots$ of $z_{n(1)}, z_{n(2)}, z_{n(3)}, \ldots$ which has a limit, $z_{0}$. Since $f$ is continuous and $f\left(z_{n(i(j))}\right)=p$ for $j=1,2,3, \ldots, f\left(z_{0}\right)=p$. Since $K_{n(1)}$, $K_{n(2)}, K_{n(3)}, \ldots$ has sequential limiting set $K_{0}, z_{0}$ is in $K_{0}$. Suppose $t_{0}$ is in $K_{0}$. Then there is a sequence of points $t_{1}, t_{2}, t_{3}, \ldots$, with limit $t_{0}$, such that $t_{j}$ is in $K_{n(j)}$ for $j=1,2,3, \ldots$. Since $C$ is closed, $f$ is continuous and $f\left(t_{j}\right)$ is in $C$ for $j=1,2,3, \ldots$, $f\left(t_{0}\right)$ is in $C$. Hence $t_{0}$ is in $f^{-1}(C)$. Therefore $K_{0} \subset f^{-1}(C)$. Let $H$ be the component of $f^{-1}(C)$ containing $z_{0}$. Since $f\left(z_{0}\right)=p, H$ is in $\mathcal{H}_{p}$. Since $z_{0}$ is in $K_{0}, K_{0} \subset f^{-1}(C)$, and $K_{0}$ is a continuum, it follows that $K_{0} \subset H$, and hence $y$ is in $H$. Thus $y$ is in $A=\mathcal{H}_{p}^{*}$. Therefore $A$ is closed. Likewise $B$ is closed.

Now, since $A$ and $B$ are closed and mutually exclusive and $f^{-1}(C)$ is closed, there exist mutually exclusive open subsets of $X, U_{A}$ and $U_{B}$ such that $A \subset U_{A}, B \subset U_{B}$, and $f^{-1}(C) \subset U_{A} \cup U_{B}$. Let $s$ and $t$ be points of $X$ such that $f(s)=p$ and $f(t)=q$ ( $s$ is in $A, t$ is in $B$ ). Let

$$
Y=\left(\left(X-U_{A}\right) \times\{s\}\right) \cup\left(\left(X-\left(U_{A} \cup U_{B}\right)\right) \times f^{-1}(C)\right) \cup\left(\left(X-U_{B}\right) \times\{t\}\right) .
$$

Note that $Y$ is the union of three closed subsets of $X \times X$ and is therefore closed and hence compact. Let $Z=f \times f(Y)$. Since $Y$ is compact and $f \times f$ is continuous, $Z$ is compact. 
We now show that $(p, q)$ is in $Z,(q, p)$ is in $Z$ and that $Z$ misses the diagonal, $\Delta_{M}$ of $M \times M$. Since $s$ is in the $A, s$ is in $X-U_{B}$, thus $(t, s)$ is in $Y$ and therefore $(f(s), f(t))=(p, q)$ is in $Z$. Since $t$ is in $B, t$ is in $X-U_{A}$, thus $(t, s)$ is in $Y$ and hence $(f(t), f(s))=(q, p)$ is in $Z$. Suppose $(x, s)$ is in $\left(X-U_{A}\right) \times\{s\}$. Then $x$ is not in $A$, hence $f(x) \neq p$. But $f(s)=p$ so $(f(x), f(s))$ is not in $\Delta_{M}$. Likewise, if $(x, t)$ is in $\left(X-U_{B}\right) \times\{t\}$ then $(f(x), f(t))$ is not in $\Delta_{M}$. Suppose $(x, y)$ is in $\left(X-\left(U_{A} \cup U_{B}\right)\right) \times f^{-1}(C)$. Then $x$ is not in $f^{-1}(C)$ since $f^{-1}(C) \subset U_{A} \cup U_{B}$. Since $y$ is in $f^{-1}(C), f(x) \neq f(y)$. Therefore $(f(x), f(y))$ is not in $\Delta_{M}$. Hence $Z$ and $\Delta_{M}$ are mutually exclusive.

We now show that $Z$ is a continuum by showing that it is connected. Suppose it is not connected. Then there exist mutually exclusive open subsets of $M \times M, V_{1}$ and $V_{2}$, such that $Z \subset V_{1} \cup V_{2}$ and each of $V_{1}$ and $V_{2}$ contains a point of $Z$. Suppose $x$ is in $X$. Then either $x$ is in $U_{A}, x$ is in $U_{B}$, or $x$ is in $X-\left(U_{A} \cup U_{B}\right)$.

Suppose $x$ is in $U_{A}$. Then $(x, t)$ is the only point in $Y$ with first coordinate $x$. Let $i(x)$ be the integer in $\{1,2\}$ such that $f \times f(x, t)$ is in $V_{i(x)}$. There exist open subsets $G_{x}$ and $R_{x}$ of $X$ such that $x$ is in $G_{x}, t$ is in $R_{x}, G_{x} \subset U_{A}$ and $f \times f\left(G_{x} \times R_{x}\right) \subset V_{i(x)}$. If $\left(x^{\prime}, w\right)$ is in $Y \cap\left(G_{x} \times X\right)$ then $x^{\prime}$ is in $G_{x} \subset U_{A}$ and hence $w=t$. Thus $\left(x^{\prime}, w\right)$ is in $G_{x} \times R_{x}$. Hence there exist an open subset $G_{x}$ of $X$ and an integer $i(x)$ in $\{1,2\}$ such that $x$ is in $G_{x}$ and $f \times f\left(\left(G_{x} \times X\right) \cap Y\right) \subset V_{i(x)}$. Likewise, for $x$ in $U_{B}$ there exist an open subset $G_{x}$ of $X$ and an integer $i(x)$ in $\{1,2\}$ such that $x$ is in $G_{x}$ and $f \times f\left(\left(G_{x} \times X\right) \cap Y\right) \subset V_{i(x)}$.

Suppose $x$ is in $X-\left(U_{A} \cup U_{B}\right)$. Since $f \times f\left(\{x\} \times f^{-1}(C)\right)=\{f(x)\} \times C$ is a continuum, $\{x\} \times f^{-1}(C) \subset(f \times f)^{-1}\left(V_{i}\right)$ for $i=1$ or $i=2$. Denote this integer by $i(x)$. By [6, Theorem 12, p. 142] there exist open subsets of $X, G_{x}$ and $R_{x}$ such that $x$ is in $G_{x}, f^{-1}(C) \subset R_{x}$ and $G_{x} \times R_{x} \subset(f \times f)^{-1}\left(V_{i(x)}\right)$. If $\left(x^{\prime}, w\right)$ is in $\left(G_{x} \times X\right) \cap Y$ then either $x^{\prime}$ is in $X-\left(U_{A} \cup U_{B}\right)$ and $w$ is in $f^{-1}(C)$ or $w=s$ or $w=t$. In the last two cases $w$ is in $f^{-1}(C)$ also. Thus $\left(G_{x} \times X\right) \cap Y \subset G_{x} \times R_{x}$ and

$$
f \times f\left(\left(G_{x} \times X\right) \cap Y\right) \subset f \times f\left(G_{x} \times R_{x}\right) \subset V_{i(x)} .
$$

In all of the three cases, $x$ in $U_{A}, x$ in $U_{B}$, and $x$ in $X-\left(U_{A} \cup U_{B}\right)$, there is a $w$ in $X$ such that $(x, w)$ is in $Y$. Hence, for each $x$ in $X$, there is an integer $i(x)$ in $\{1,2\}$ and an open set $G_{x}$ containing $x$ such that $f \times f\left(\left(G_{x} \times X\right) \cap Y\right) \subset V_{i(x)}$.

Since $V_{1}$ intersects $Z$, there is a point $(x, w)$ in $Y$ such that $f \times f(x, w)$ is in $V_{1}$. Therefore there is a point $x$ in $X$ such that $i(x)=1$. Similarly, there is a point $x$ in $X$ such that $i(x)=2$. Let

$$
W_{1}=\left\{G_{x}: i(x)=1\right\}^{*} \text { and } W_{2}=\left\{G_{x}: i(x)=2\right\}^{*} .
$$

The sets $W_{1}$ and $W_{2}$ are open.

Suppose $r$ is in $W_{1} \cap W_{2}$. Then there is a point $x_{1}$ in $X$ such that $r$ is in $G_{x_{1}}$ and $i\left(x_{1}\right)=1$ and a point $x_{2}$ in $X$ such that $r$ is in $G_{x_{2}}$ and $i\left(x_{2}\right)=2$. Let $y$ be a point in $X$ such that $(r, y)$ is in $Y$. Now $f \times f(r, y)$ is in $V_{j}$ since $f \times f\left(\left(G_{x,} \times X\right) \cap Y\right) \subset V_{j}$, for $j=1,2$. This is inconsistent with the fact that $V_{1}$ and $V_{2}$ are mutually exclusive. Hence $W_{1}$ and $W_{2}$ are mutually exclusive.

Thus $X \subset W_{1} \cup W_{2}$ where $W_{1}$ and $W_{2}$ are mutually exclusive nonempty open subsets of $X$. This is a contradiction since $X$ is a continuum. Therefore $Z$ is a 
continuum. Since $(p, q)$ is in $Z$ and $(q, p)$ is in $Z$ and $Z$ does not intersect $\Delta_{M}$, we have a contradiction with Theorem 2(3). Therefore $M$ is in class $W$.

5. A class of continua for which the span and semispan are equal. A. Lelek has given an example [9, Example 1.5, p. 37] of a continuum $X$ such that $\sigma_{0}(X)=1$ and $\sigma(X)=\frac{1}{2}$. The following theorem shows that under suitable hypothesis the span and semispan agree.

THEOREM 5. If every subcontinuum of the continuum $M$ is in class $W$, then $\sigma(M)=\sigma_{0}(M)$.

Proof. Suppose $M$ is a continuum with metric $d$ and that every subcontinuum of $M$ is in class $W$. By [9, Proposition 1.1, p. 36], $\sigma(M) \leqslant \sigma_{0}(M)$. Suppose $\varepsilon>0$ and $\sigma_{0}(M) \geqslant \varepsilon$. Let $Z$ be a subcontinuum of $M \times M$ such that $\pi_{2}(Z) \subset \pi_{1}(Z)$ and $d(x, y) \geqslant \varepsilon$ for each point $(x, y)$ in $Z$.

We choose inductively a sequence $K_{0}, K_{1}, K_{2}, \ldots$ of subcontinua of $Z$ such that for each positive integer $i$,

$$
K_{i} \subset K_{i-1}
$$

and

$$
\pi_{1}\left(K_{i}\right)=\pi_{2}\left(K_{i-1}\right) .
$$

Let $K_{0}=Z$. Suppose that $K_{i}$ has been chosen for $0 \leqslant i \leqslant n$ in such a way that (1) and (2) are satisfied. Since $K_{n} \subset K_{n-1}$, it follows that $\pi_{2}\left(K_{n}\right) \subset \pi_{2}\left(K_{n-1}\right)=\pi_{1}\left(K_{n}\right)$. Let $\rho_{n}$ be the restriction of $\pi_{1}$ to $K_{n}$. By hypotheses, $\pi_{1}\left(K_{n}\right)=\rho_{n}\left(K_{n}\right)$ is in class $W$. Thus $\rho_{n}$ is weakly confluent and there is a component $T$ of $\rho_{n}^{-1}\left(\pi_{2}\left(K_{n}\right)\right)$ such that $\pi_{1}(T)=\rho_{n}(T)=\pi_{2}\left(K_{n}\right)$. Choose one such $T$ and denote it by $K_{n+1}$. Condition (2) then holds for $i=n+1$. Condition (1) holds also because $K_{n+1} \subset \rho_{n}^{-1}\left(\pi_{2}\left(K_{n}\right)\right) \subset K_{n}$. Let $H=\bigcap_{i>0} K_{i}$. Then $H$ is a continuum. Moreover

$$
\begin{aligned}
\pi_{2}(H) & =\pi_{2}\left(\bigcap_{i>0} K_{i}\right)=\bigcap_{i>0} \pi_{2}\left(K_{i}\right)=\bigcap_{i>0} \pi_{1}\left(K_{i+1}\right) \\
& =\pi_{1}\left(\bigcap_{i>0} K_{i+1}\right)=\pi_{1}(H) .
\end{aligned}
$$

If $(x, y)$ is in $H$ then $(x, y)$ is in $Z$ and hence $d(x, y) \geqslant \varepsilon$. Therefore $\sigma(M) \geqslant \varepsilon$. Thus $\sigma(M) \geqslant \sigma_{0}(M)$, and therefore $\sigma(M)=\sigma_{0}(M)$.

6. The equivalence of zero span and zero semispan. We now apply Theorem 5 to prove the principal result of this paper.

THEOREM 6. If $M$ is a continuum then $\sigma(M)=0$ if and only if $\sigma_{0}(M)=0$.

Proof. Suppose $M$ is a continuum. If $\sigma_{0}(M)=0$ then $\sigma(M)=0$ by [9, Proposition 1.1, p. 36].

Suppose $\sigma(M)=0$. If $H$ is a subcontinuum of $M$ then $\sigma(H)=0$ and hence $s(M)=0$ by Theorem 1 . Thus $H$ is in class $W$ by Theorem 4 . Therefore, $\sigma_{0}(M)=$ $\sigma(M)=0$ by Theorem 5 . 
COROllary. If $M$ is a continuum and $\sigma(M)=0$ then $M$ has the incidence point property and $M$ has the fixed point property.

Proof. Suppose $M$ is a continuum, $f$ is a mapping of $X$ onto $M$ and $g$ is a mapping of $X$ into $M$. Let $Z=\{(f(x), g(x)): x \in X\}$. Then $Z$ is a subcontinuum of $M \times M$ and $\pi_{2}(Z) \subset M=\pi_{1}(Z)$. Since $\sigma(M)=0, \sigma_{0}(M)=0$ by Theorem 6 and thus $Z$ intersects $\Delta_{M}$, that is, there is a point $x$ of $X$ such that $f(x)=g(x)$. Hence $M$ has the incidence point property. By letting $X=M$ and $f=\mathrm{id}_{M}$, we see that $M$ also has the fixed point property.

7. A sum theorem for continua with zero span. Fugate [2, Theorem 1, p. 466] proved that if $H$ and $K$ are chainable continua which intersect, then $H \cup K$ is chainable if and only if $H \cup K$ is atriodic and unicoherent. Ingram [4, Theorem 2, p. 196] strengthened this to the following: If $H$ and $K$ are chainable continua which intersect, then $H \cup K$ is chainable if and only if $H \cup K$ is atriodic and $H \cap K$ is connected. In [1] Edwin Duda and James Kell prove that this statement remains true if chainable is replaced by zero semispan. We now apply Theorem 6 and the result by Duda and Kell to prove the following:

THEOREM 7. If $H$ and $K$ are continua which intersect, $\sigma(H)=0, \sigma(K)=0, H \cup K$ is atriodic, and $H \cap K$ is connected, then $\sigma(H \cup K)=0$.

Proof. Suppose that $H$ and $K$ are continua which intersect, $\sigma(H)=0, \sigma(K)=0$, $H \cup K$ is atriodic, and $H \cap K$ is connected. Then $\sigma_{0}(H)=0$ and $\sigma_{0}(K)=0$ by Theorem 6. Thus by [1, Theorem 3.2], it follows that $\sigma_{0}(H \cup K)=0$. Therefore $\sigma(H \cup K)=0$, again by Theorem 6 .

Acknowledgements. The author wishes to thank Professor W. T. Ingram for many helpful discussions concerning the results in this paper, and Professor A. Lelek who pointed out that the argument originally given for Theorem 6 actually proves the stronger Theorem 5. The author would also like to thank the referee for his careful reading of the paper and for suggestions which helped to shorten it.

\section{REFERENCES}

1. E. Duda and J. Kell III, Two sum theorems for semispan, Houston J. Math. 8 (1982), 317-321.

2. J. B. Fugate, Decomposable chainable continua, Trans. Amer. Math. Soc. 123 (1966), 460-468.

3. W. T. Ingram, An atriodic tree-like continuum with positive span, Fund. Math. 77 (1972), 99-107.

4. __ Decomposable circle-like continua, Fund. Math. 63 (1968), 193-198.

5. Atriodic tree-like continua and the span of mappings, Topology Proc. 1 (1976), 329-333.

6. J. L. Kelley, General topology, Van Nostrand, Princeton, N. J., 1960.

7. A. Lelek, Disjoint mappings and the span of spaces, Fund. Math. 55 (1964), 199-214.

8. __ Some problems concerning curves, Colloq. Math. 23 (1971), 93-98.

9. __ On the surjective span and semispan of connected metric spaces, Colloq. Math. 37 (1977), 35-45.

10. R. L. Moore, Foundations of point set theory, Amer. Math. Soc. Colloq. Publ., vol. 13, Amer. Math. Soc., Providence, R. I., 1962.

11. R. H. Sorgenfrey, Concerning triodic continua, Amer. J. Math. 66 (1944), 439-460.

Department of Mathematics, California State University, Sacramento, California 95819

Current address: Texaco Bellaire Research Laboratories, Bellaire, Texas 77401 